\title{
The Orchid MADS-Box Genes Controlling Floral Morphogenesis
}

\author{
Wen-Chieh Tsai $^{1}$ and Hong-Hwa Chen ${ }^{1,2, *}$ \\ ${ }^{1}$ Department of Life Sciences and ${ }^{2}$ Institute of Biotechnology, National Cheng Kung \\ University, Tainan 701, Taiwan \\ E-mail: hhchen@mail.ncku.edu.tw
}

Received May 15, 2006; Accepted June 29, 2006; Published July 14, 2006

Orchids are known for both their floral diversity and ecological strategies. The versatility and specialization in orchid floral morphology, structure, and physiological properties have fascinated botanists for centuries. In floral studies, MADS-box genes contributing to the now famous ABCDE model of floral organ identity control have dominated conceptual thinking. The sophisticated orchid floral organization offers an opportunity to discover new variant genes and different levels of complexity to the ABCDE model. Recently, several remarkable research studies done on orchid MADS-box genes have revealed the important roles on orchid floral development. Knowledge about MADS-box genes' encoding ABCDE functions in orchids will give insights into the highly evolved floral morphogenetic networks of orchids.

KEYWORDS: orchids, MADS-box genes, ABCDE model, floral development, floral morphogenetic networks

\section{INTRODUCTION}

With more than 270,000 known species, angiosperms are by far the most diverse and widespread group of plants. The ancestry of the angiosperm is still uncertain. The fossil records show that the angiosperms appear at the early Cretaceous period, about 130 million years ago. By the end of the Cretaceous, 65 million years ago, the angiosperms had radiated and become the dominant plants on Earth, as they are today. The origin and diversification of angiosperms, what Charles Darwin characterized as "an abominable mystery", has been the subject of much speculation for the last 100 years[1,2,3]. The rapid explosion in diversity that followed their origin in the early Cretaceous may be linked to modularity within their new structure, the flower[4]. The flower is the defining reproductive adaptation of angiosperms, and is the predominant source of characters for angiosperm taxonomy and phylogeny reconstruction[5].

Over the past decades, the codification of rigorous methods of phylogenetic analysis, the emergence of molecular techniques, and a renewed interest in the developmental pathways followed during the growth of plant organs have improved understanding of angiosperm relationships[6,7,8,9]. The angiosperms consist of some small relic basal clades (basal angiosperms), magnollids, and two main clades: monocots and eudicots (Fig. 1). The basal angiosperms and magnollids share some primitive traits, such as a typical spiral rather than whorled arrangement of flower organs[10,11]. The monocots show extreme variation in floral form, 


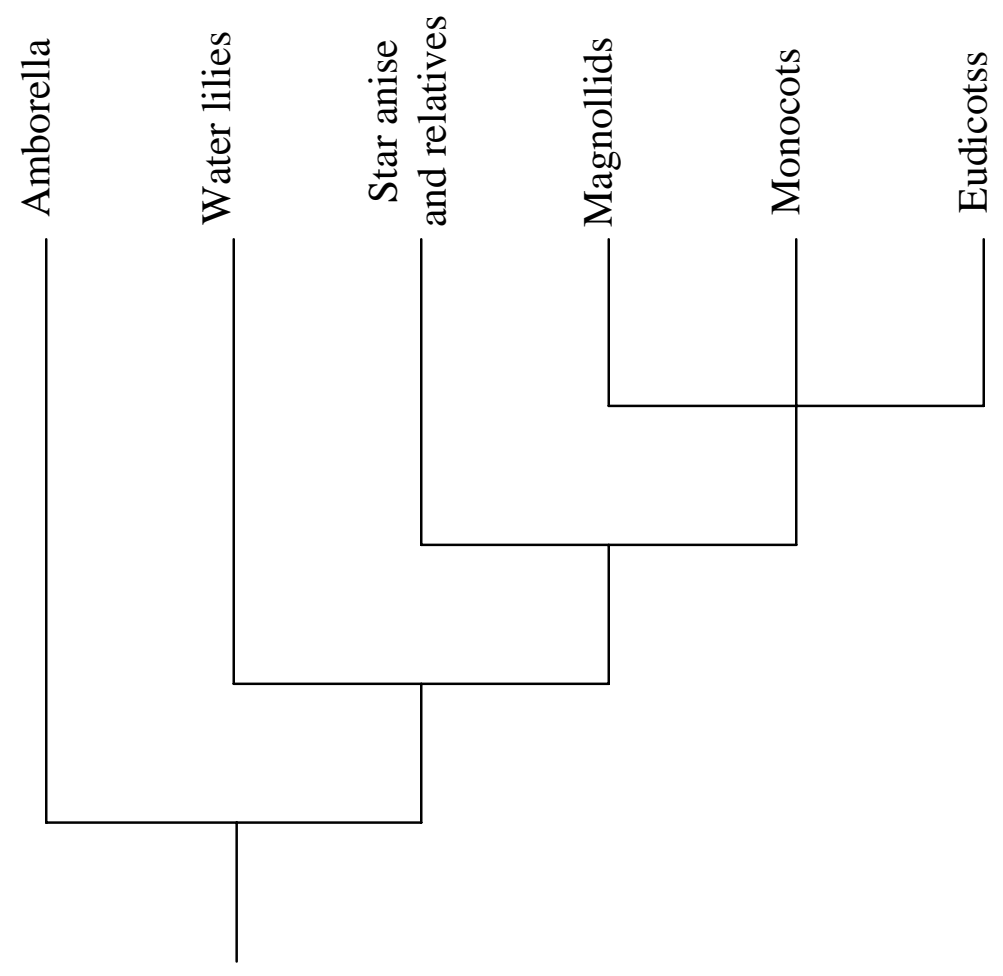

FIGURE 1. Phylogeny of angiosperms.

including bilaterally symmetric flowers with elaborately modified perianth parts. The organization of flower parts is a bit less variable in the core eudicots.

For plants, the MADS-box-containing transcriptional regulators have been the focus of floral organ specification, development, and evolutionary studies[12,13,14]. In the well-known "ABC model”, the organ identity in each whorl is determined by a unique combination of three activities by organ identity genes, called A, B, and C[12]. In any one of the four flower whorls, expression of A alone specifies sepal formation. The combination of $\mathrm{AB}$ determines the development of petals and the combination of $\mathrm{BC}$ offers stamen formation. Expression of the $\mathrm{C}$ function alone determines the development of carpels. Functions of $\mathrm{A}$ and $\mathrm{C}$ are mutually repressive[15]. The ABC genes were cloned from a wide range of species and the model has been used to explain floral organ development in plants[15,16,17,18,19,20,21]. On the basis of studies on Petunia, the "ABC model" was later extended by including D-class genes that specify ovules[22]. An important recent discovery was that another set of MADS-box genes, SEPALLATA1, 2, and 3, function redundantly to specify petals, stamens, and carpels as well as floral determinacy[23]. Recently, SEP4 has been defined. The sep1 sep2 sep3 sep4 quadruple mutants develop vegetative leaves rather than sepals, petal, stamens, or carples[24]. SEPALLATA function, or so-called E function, has led to a revision of the "ABC model"' to become the "ABCDE model"[25,26]. The diversification of MADS-box genes during evolution has been proposed to be a major driving force for floral diversity in land plant architecture[15,27].

Containing more than 20,000 species, the Orchidaceae, classified in class Liliopsida, order Asparagales, is one of the largest angiosperm families. Associated with this enormous size is an extraordinary floral diversity. Orchids are extremely rich in species and speciation rates are presumed to be exceptionally high[28]. Although it has often been hypothesized that this spectacular diversification is linked to the intimate and sometimes bizarre interaction of many species with their pollinators[29], we are still facing the challenge of explaining how these mechanisms work and why they have evolved. 
According to the classic view, the orchid flower is composed of five whorls of three segments each including two perianth whorls, two staminal whorls, and one carpel whorl (Fig. 2A)[30]. This also conforms to the general flower structure of many other monocotyledonous families. Orchidaceae represent an unusually coherent group among monocots, possessing several reliable floral morphological synapomorphies, including the presence of a gynostemium, or column, fused by the style and at least part of the androecium, a highly evolved petal called labellum, and resupination caused by $180^{\circ}$ torsion of the pedicel[31]. Within the monocots, only well-known crop species, such as rice and maize, have been studied thoroughly, but the highly reduced flowers make them unsuitable for general floral development studies. All expected whorls in the flowers are present in orchids, and the highly sophisticated flower organization offers an opportunity to discover new variant genes and different levels of complexity within morphogenetic networks. Thus, the Orchidaceae can be used to test the validity of the "ABC model" in the monocots and to study how MADS-box genes are involved in defining the different highly specialized structures in orchid flowers.

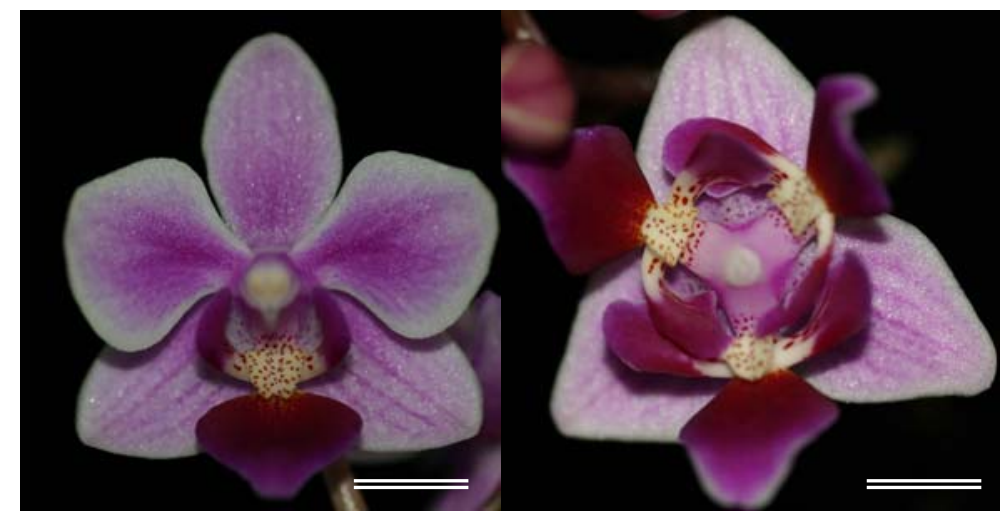

A

B

FIGURE 2. Flowers from wild-type (A) and peloric mutant (B) of Phalaenopsis equestris. Bar $=1 \mathrm{~cm}$.

\section{A-CLASS GENES IN ORCHIDS}

To date, almost all cloned orchid MADS-box genes involved in floral development were from Epidendroideae, the largest orchid subfamily with many more genera and species than all of the other four subfamilies together (Table 1). So far, four A-class genes were identified from Dendrobium. One of them was isolated from Dendrobium grex Madame Thong-In, named as DOMADS2[32]. The other three genes were cloned from D. thyrsiflorum, named as DthyrFL1, DthyrFL2, and DthyrFL3, respectively[33]. The APETALA1/FRUITFULL (AP1/FUL) MADS-box gene lineage within the eudicots was recognized as two clades (the euAP1 and euFUL clade), whereas the noncore eudicots and monocots only have sequences similar to euFUL genes[34]. Sequence analysis showed that these genes contain the C-terminal FUL-like motif LPPWML of monocot FUL-like proteins, but this motif is not present in the sequence of DthyrFL3[33]. Phylogenetic analysis showed that DthyrFL1 and DOMADS2 are orthologous genes (Fig. 3A). The existence of DthyrFL2 and DthyrFL3 represent a recent duplication event in D. thyrsiflorum (Fig. 3A[33]). DOMADS2 is expressed in both of the shoot apical meristem and the emerging floral primordium throughout the process of floral transition and later in the column of mature flowers[32]. The expression pattern of DOMADS2, from shoot apical meristem and increasing in later stages of floral development, suggests that DOMADS2 is one of the earliest regulatory genes during the transition of flowering. DthyrFL genes are expressed not only during inflorescence development, but also in developing ovules[33]. These A-class genes in orchids may be involved in floral meristem identity, and in 
TABLE 1

Floral MADS-Box Genes and Their Expression Patterns in Orchidaceae

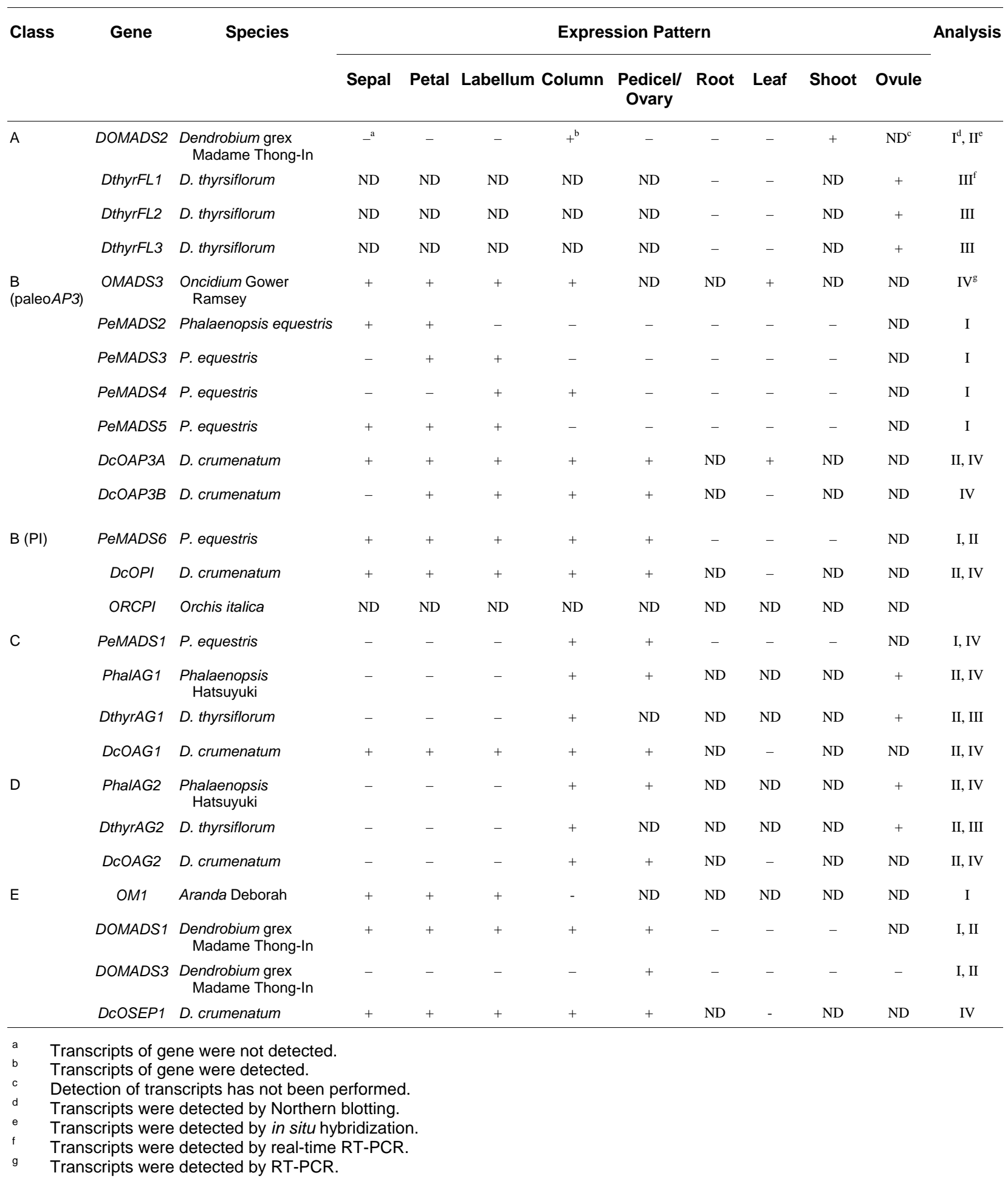




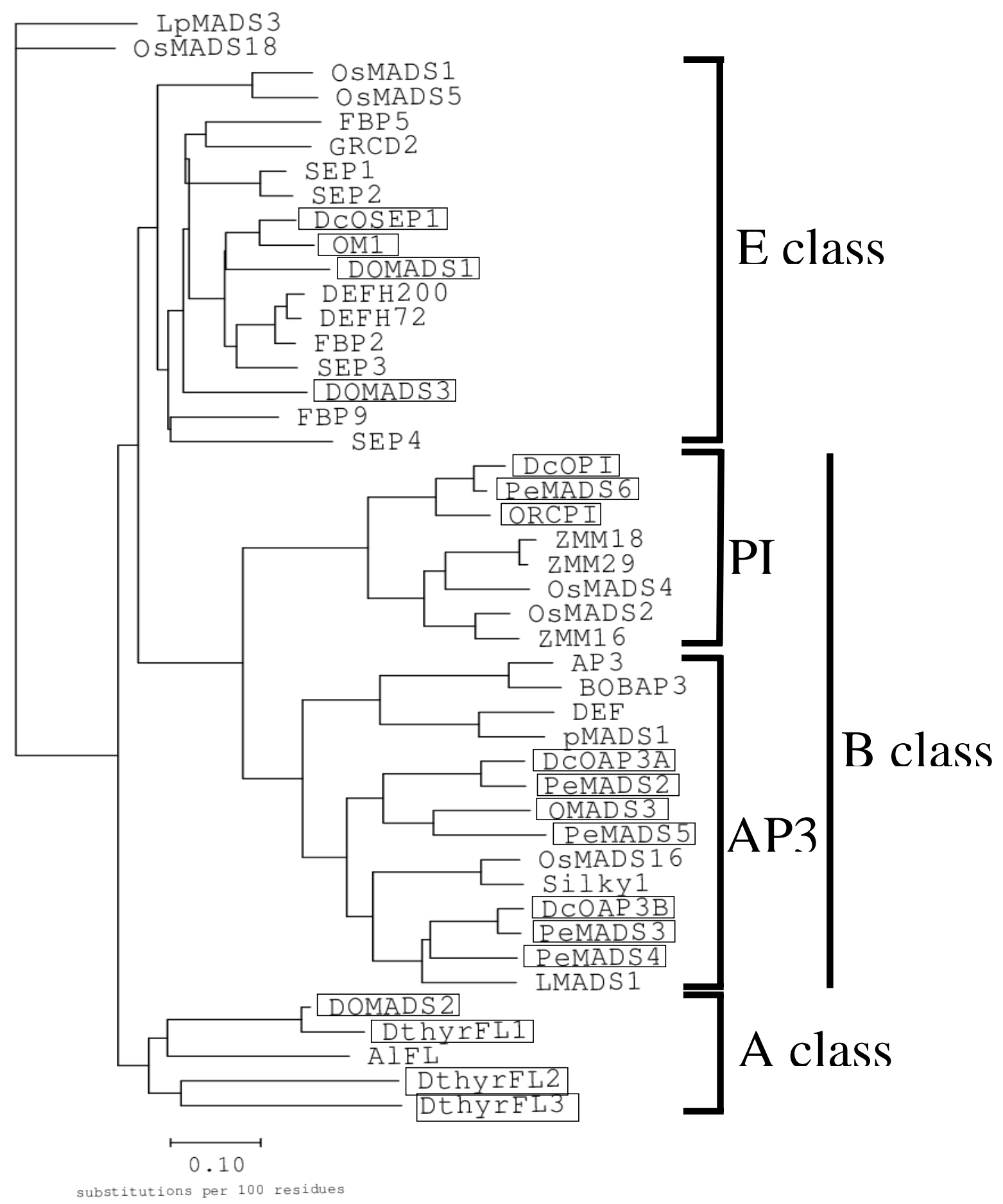

A

FIGURE 3. Phylogenetic relationship of MADS-box genes of ABCDE class. (A) Phylogenetic analysis of A-, B-, and E-class genes. (B) Phylogenetic analysis of C- and D-class genes. Orchid MADS-box genes are highlighted by open boxes. 


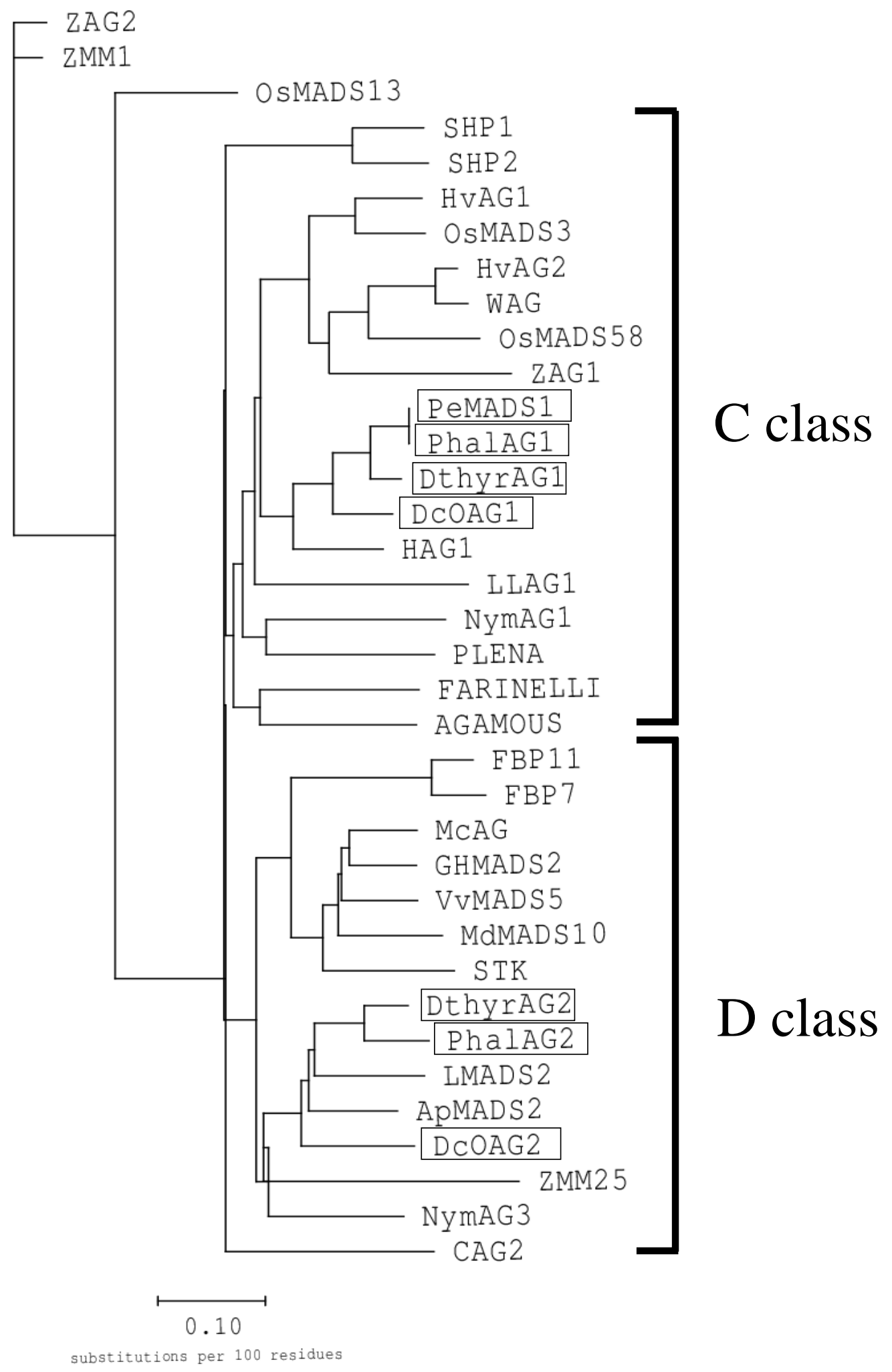

FIGURE 3B 
column and ovule development. Unlike its homolog AP1 in Arabidopsis, the DOMADS2 may not correlate with the development of the first two whorls. However, it is not clear whether the DthyrFL genes associate with perianth formation. In addition, a MADS-box gene OMADS1 in the Oncidium orchid, also involved in floral initiation and formation, belongs to the AGL6 subfamily rather than the Aclass gene[35]. Transgenic Arabidopsis and tobacco overexpressing OMADS1 showed significantly reducing plant size, extremely early flowering, and losing inflorescence indeterminacy[35].

\section{B-CLASS GENES IN ORCHIDS}

Both the developmental and the biochemical aspects of B-class genes required to specify the identity of petals in whorl 2 and stamens in whorl 3 appear to be conserved in many core eudicots[15,16]. The Bclass genes in monocots rice and maize are similar in function to the core eudicot B-class genes[19,20,36,37]. The orchid flowers have a petaloid perianth arrangement that could be explained by a "modified ABC model" in that the expression of the B-class genes has expanded to whorl 1[38]. In addition, the orchid flowers display an elaborated labellum that is a highly modified petal. As the function of A-class genes is poorly defined in angiosperms, studies of petal development and evolution have generally focused on B-class genes[39]. The extraordinary floral diversity in orchids may be correlated with the evolution of B-class genes.

Molecular functions of B-class genes were studied in more detail than any other floral homeotic genes in orchids. So far, various numbers of APETALA3 (AP3)-like and PISTILLATA (PI)-like genes have been isolated from several orchids. These include one AP3-like OMADS3 isolated from Oncidium Gower Ramsey, four AP3- and one PI-like genes identified from Phalaenopsis equestris, and two AP3- and one $P I$-like genes cloned from Dendrobium crumenatum[40,41,42,43]. All these AP3-like genes in orchids are the members of the paleoAP3 lineage (Fig. 3A). The paleoAP3 genes identified from orchids were subdivided into two subclades. One subclade contains OMADS3, PeMADS5, DcOAP3A, and PeMADS2, while OcOAP3B, PeMADS3, and PeMADS4 make up the other subclade (Fig. 3A). This result suggests that the ancestor of Orchidaceae might have had two paleoAP3-like genes, and further gene duplication has at least taken place in the AP3 clade in the monocots. Interestingly, both OMADS3 and PeMADS5 do not present obvious paleoAP3 motif, suggesting that they are orthologous genes. Although they share similar expression patterns in orchid floral organs, PeMADS5 is not expressed in vegetative tissues, but the expression of OMADS3 can be detected in leaves[40,41]. Phylogenetic analysis also showed that DcOAP3A and PeMADS2 are orthologous genes (Fig. 3A). However, they possess different expression patterns. Similar to OMADS3, the DCOAP3A is ubiquitously expressed in all floral organs and in leaves, while PeMADS2 is predominantly expressed in sepals and petals[40,41,43]. Recently, we discovered that there were at least three paleo $A P 3$ genes displaying distinct expression patterns in Oncidium floral organs (our unpublished data). In addition, we also noticed that the expression profile of OMADS3 examined by Hsu and Yang[40] was indeed composed of the expression patterns from two paleoAP3 genes of Oncidium (our unpublished data). Distinctly, a specialized paleoAP3 gene, PeMADS4 discovered in $P$. equestris, is specifically expressed in labellum and column, suggesting an association of its function with the development of orchid labellum and column. Gene duplication is important for generating new genes during evolution[44], and thus may lead to the generation of new organs. In orchids, duplication of paleoAP3-like genes, followed by diversification and specialization probably is associated concomitantly with the arising of new floral organ, labellum, in orchids.

Overexpression of paleoAP3 genes from Oncidium, Dendrobium, and Phalaenopsis under the control of the cauliflower mosaic virus 35S promoter was examined in Arabidopsis[40,43,our unpublished data]. Consistently, all these results showed that the flower morphology of the transgenic Arabidopsis plants overexpressing the orchid paleoAP3 genes are indistinguishable from that of the wild-type plants. Dominant negative mutation strategy was further conducted to investigate the functions of OMADS3 and $D c O A P 3 A[43]$. By doing this, the OMADS3 has been shown to have a function similar to the A functional gene in regulating flower formation as well as floral initiation, whereas the DcOAP3A has a putative $\mathrm{B}$ 
function[40,43]. However, these results could not reflect the real roles they may play during orchid floral development.

The peloric flowers that are actinomorphic mutants with lip-like petals are widely found in natural populations of species from Veronicaceae, Gesneriaceae, Labiatae, and Orchidaceae[45]. With the presence of a high frequency of orchid peloric mutants derived from micropropagation (Fig. 2B), we were able to infer the individual roles played by the diversified paleoAP3 genes in orchids by comparing the expression patterns of the four paleoAP3 (PeMADS2, PeMADS3, PeMADS4, and PeMADS5) genes in wild-type Phalaenopsis floral organs and in peloric mutants[41]. First, we discovered that both PeMADS2 and PeMADS5 were expressed in the sepal of wild-type plants, but only PeMADS2 transcript was detected in the sepal of the peloric mutant whose morphology is not affected. This result suggests that PeMADS5 is dispensable, while PeMADS2 is crucial for the sepal development. Second, the expressions of all four PeMADS genes, except PeMADS4, were detected in the wild-type petals. However, the expression of PeMADS5 was not noted in the lip-like petals of the peloric mutant. This result suggests that PeMADS5 correlates with the petal development. Third, the expression of PeMADS4 is concentrated in lips and columns in the wild-type plant, and is extended to the lip-like petals in the peloric mutant. The fact that the PeMADS4 transcript was detected in the lip-like petal of the peloric mutant suggests that PeMADS4 is required for labellum identity. Fourth, the PeMADS3 shows similar expression patterns in the wild-type plant and the peloric mutant, suggesting its important function in inner perianth whorl morphogenesis.

So far, there is only one $P I$-like gene found in $D$. crumenatum and $P$. equestris, $D c O P I$ and PeMADS6, respectively[42,43]. The Southern blot hybridization results supported that the Phalaenopsis orchid genome contains only one copy of the PI-like gene[42]. Both genes are expressed in all floral organs, except that PeMADS6 is not detected in the pollinia of $P$. equestris[42,43]. In addition, the PeMADS6 is expressed in the undeveloped ovary[42]. Tsai et al.[42] suggested that the expression of PeMADS6 in ovary has an inhibitory effect on the development of the ovary, and auxin acts as the candidate signal to regulate the repression of PeMADS6 expression in the ovary. Furthermore, the PeMADS6 is not differentially expressed between wild-type and peloric floral organs, which suggests that PeMADS6 is not responsible for the altered phenotype of the peloric mutant. Overexpression of DcOPI or PeMADS6 in Arabidopsis demonstrated that both of them share the angiosperm PI function[42,43]. Further evidence came from the complementation of pi-1 phenotype in Arabidopsis by overexpressing DcOPI and showed that DcOPI is able to substitute PI in Arabidopsis[42], while PeMADS6 could not complement pi-4 mutant (our unpublished data).

In conclusion, expression patterns of B-class genes in orchid floral organs nicely fit the "modified ABC model" in that the expression of the B-class genes has expanded to whorl 1 in plants possessing nearly identical morphology of sepals and petals[38]. paleoAP3 genes are highly duplicated in Epidendroideae genome. Diversification and fixation of both these gene sequences and expression profiles might cause the subfunctionalization and even neofunctionalization. Driving force of specialized labellum and diversified orchid flowers may be linked to the fast evolution rate of paleoAP3 genes. Study of the B-class genes from other orchid subfamilies, such as Apostasioideae, Cypripedioideae, Spiranthoideae, Orchidoideae, and even more members of Epidendroideae[46], will provide profound knowledge to resolve.

\section{C- AND D-CLASS GENES IN ORCHIDS}

A gynostemium or column, comprising stamen filaments adnate to a syncarpous style, is normally regarded as a structure peculiar to the orchids[46]. The development of a column, which involves whorl 3 and whorl 4, would be one of the most interesting subjects to elucidate the evolution of C-class genes. In most orchid flowers, ovary and ovule development is precisely and completely triggered by pollination, and thus orchids offer a unique opportunity to study D-class genes involving ovule development. More recently, one C-class gene and one D-class gene were isolated independently from three orchid species, 
Phalaenopsis Hatsuyuki (PhalAG1, PhalAG2[47]), D. thyrsiflorum (DthyrAG1, DthyrAG2[48]), and D. crumenatum (DcOAG1, DcOAG2[43]). PhalAG1, DthyrAG1, DcOAG1 were classified in C-lineage of AG-like genes, and PhalAG2, DthyrAG2, DcOAG2 were classified in D-lineage of AG-like genes (Fig. 3B). The PhalAG1, PhalAG2, DthyrAG1, and DthyrAG2 share very similar spatial expression patterns in column, ovary, and developing ovules despite the fact that these four genes belong to different lineages[47,48]. One possible explanation is that C- and D-class genes in orchids would act redundantly with each other in floral and ovule development. Although PhalAG1 is expressed in all floral organs at their initiation, its expression quickly decreases and then can only be detected in column and ovary when flowers mature, and this is also true for DthyrAG1[47,48]. We also identified a C-class gene, PeMADS1, from P. equestris, and expression patterns investigated were consistent with PhalAG1 and DthyrAG1 (our unpublished data). However, DcOAG1 is expressed in all mature floral organs and DcOAG2 is expressed in anther cap and column of D. crumenatum[43]. The unusual expression patterns of DcOAG1 in monocots evoke that the regulatory mechanism of DcOAG1 is independently evolved in D. crumenatum as some basal angiosperms, such as Illicium and Persea[49], but the function of DcOAG1 and Arabidopsis $A G$ is conserved as supported by the phenotypic similarity between transgenic Arabidopsis expressing either 35S::DcOAG1 or 35S::AG[43]. Molecular mechanism of morphogenesis of orchid gynostemium is still enigmatic. Mutation of C-class genes in orchids could possibly provide the opportunity to shed light on the mystery.

\section{E-CLASS GENES IN ORCHID}

E-class genes are required for floral organ identity in all four floral organs as well as floral determinacy[24,50]. They have been shown to form ternary complexes with A-class proteins and with Bclass proteins in yeast three-hybrid system and can mediate the interactions between B- and C-class proteins in higher-order complexes[51]. The first E-class gene, OM1, was isolated from the supposed bigeneric hybrid Aranda Deborah[52]. The other three E-class genes (DOMADS1, DOMADS3, DCOSEP1) were identified from Dendrobium; two of them were cloned from Dendrobium grex Madame Thong-In, the other one was isolated from D. crumenatum[32,43]. Phylogenetic analysis showed that OM1 was clustered with DOMADS1 and DcOSEP1, and DOMADS3 separated itself from the other three orchid E-class genes at a distance (Fig. 3A). DOMADS1 RNA is uniformly expressed in both of the inflorescence meristem and floral primordium and later existed in all of the floral organs[32]. The expression pattern of DOMADS1 in mature flowers coincides with its counterpart DcOSEP1 in D. crumenatum, as with their orthologs in Arabidopsis[23,32,43]. However, OM1 is expressed in mature flowers and not in young developing inflorescence or young floral buds. In the mature flowers, it is only expressed in petals and weakly in sepals, but not in the column[52]. Spatiotemporal expression differences imply that functional diversification among these genes closely relates in phylogeny. The onset of DOMADS3 transcription is in early shoot apical meristem at the stage before the differentiation of the first flower primordium, and later can only be detected in the pedicels[32]. The DOMADS3 may function as a regulatory factor not only in early floral transition, but also in the development of the pedicel. The expression of E-class genes overlapping with ABC genes in orchids suggests that the higherorder MADS complexes are involved in orchid floral development. Recently, one line of evidence that MADS proteins form higher-order complexes comes from the formation of DcOAP3A-DcOPI-DcOSEP1 and DcOAP3B-DcOPI-DcOSEP1 was detected by using yeast three-hybrid experiments[43].

\section{PERSPECTIVE}

Owing to the large genome size, long life cycle, and inefficient transformation system of orchids, a few studies for orchid biology exist. Recently, the genetic architecture of orchid-sophisticated floral organization has begun to be investigated. Given that the orchids represent the most successful and 
diverse plant families worldwide, the development of genomic resources is an imperative[53]. Thank to the advanced progress of genomics and bioinformatics, plentiful gene information and integrated bioinformatic tools are ready to be used for studying orchid biology[54,55,56]. The effort of many scientists will promise to lead to a better understanding of the molecular and genetic mechanisms of orchid floral control in the years to come.

\section{ACKNOWLEDGMENT}

We thank Wen-Huei Chen (Department of Life Sciences, National Science Council, National University of Kaohsiung, Kaohsiung, Taiwan) for leading us into the field of orchids. We thank Chang-Sheng Kuoh (Department of Life Sciences, National Cheng Kung University, Tainan, Taiwan) for sharing his knowledge of orchid floral ontogeny. We thank Tung-Hua David Ho (Institute of Plant and Microbial Biology, Academia Sinica, Taipei, Taiwan) and Michel Delseny (Laboratory of Plant Genome and Development, University of Perpignan, France) for helpful discussions. Grant funding from both the National Science Council and Council of Agriculture in Taiwan support the current research in our labs.

\section{REFERENCES}

1. Darwin, C. (1903) The Different Forms of Flowers on Plants of the Same Species. D. Appleton, New York.

2. Cronquist, A. (1988) The Evolution and Classification of Flowering Plants. 2nd ed. New York Botanical Garden, Bronx.

3. Doyle, J.A. (1994) Origin of the angiosperm flower: a phylogenetic perspective. Plant Sys. Evol. Suppl. 8, 7-29.

4. Carroll, S.B. (2001) Chance and necessity: the evolution of morphological complexity and diversity. Nature 409, 1102-1109.

5. Doyle, J.J. (1994) Evolution of a plant homeotic multigene family: towards connecting molecular systematics and molecular developmental genetics. Syst. Biol. 43, 307-328.

6. Crane, P.R., Friis, E.M., and Pedersen, K.R. (1995) The origin and early diversification of angiosperm. Nature 353, 31-37.

7. Soltis, P.S., Soltis, D.E., Savolainen, V., Crane, P.R., and Barraclough, T.G. (2002) Rate heterogeneity among lineages of tracheophytes: integration of molecular and fossil data and evidence for molecular living fossils. Proc. Natl. Acad. Sci. U. S. A. 99, 4430-4435.

8. Chaw, S.M., Chang, C.C., Chen, H.L., and Li, W.H. (2004) Dating the monocot-dicot divergence and the origin of core eudicots using whole chloroplast genomes. J. Mol. Evol. 58, 424-441.

9. Davies, T.J., Barraclough, T.G., Chase, M.W., Soltis, P.S., Soltis, D.E., and Savolainen, V. (2004) Darwin's abominable mystery: insights from a supertree of the angiosperms. Proc. Natl. Acad. Sci. U. S. A. 101, 1904-1909.

10. Albert, V.A., Gustafsson, M.H.G., and DiLaurenzio, L. (1998) Ontogenetic systematics, molecular developmental genetics, and the angiosperm petal. In Molecular Systematics of Plants. II. DNA Sequencing. Soltis, D.E., Soltis, P.S., and Doyle, J.J., Eds. Kluwer Academic, Boston. pp. 349-374.

11. Endress, P.K. (2001) Origins of flower morphology. J. Exp. Zool. 291, 105-115.

12. $\quad$ Weigel, D. and Meyerowitz, E.M. (1994) The ABCs of floral homeotic genes. Cell 78, 203-209.

13. Purugganan, M.D., Rounsley, S.D., Schmidt, R.J., and Yanofsky, M.F. (1995) Molecular evolution of flower development: diversification of the plant MADS-box regulatory gene family. Genetics 140, 345-356.

14. Münster, T., Pahnke, J., Di Rosa, A., Kim, J.T., Martin, W., Saedler, H., and Theissen, G. (1997) Floral homeotic genes were recruited from homologous MADS-box genes preexisting in the common ancestor of ferns and seed plants. Proc. Natl. Acad. Sci. U. S. A. 94, 2415-2420.

15. Theissen, G., Becker, A., Rosa, A.D., Kanno, A., Kim, J.T., Münster, T., Winter, K.-U., and Saedle, H. (2000) A short history of MADS-box genes in plants. Plant Mol. Biol. 42, 115-149.

16. Irish, V.F. and Kramer, E.M. (1998) Genetic and molecular analysis of angiosperm flower development. Adv. Bot. Res. 28, 197-230.

17. Kang, H.-G., Jeon, J.-S., Lee, S., and An, G. (1998) Identification of class B and class C floral organ identity genes from rice plants. Plant Mol. Biol. 38, 1021-1029.

18. Kramer, E.M., Dorit, R.L., and Irish, V.F. (1998) Molecular evolution of genes controlling petal and stamen development: duplication and divergence within the APETALA3 and PISTILLATA MADS-box gene lineage. Genetics 149, 765-783.

19. Ambrose, B.A., Lerner, D.R., Ciceri, P., Padilla, C.M., Yanofsky, M.F., and Schmidt, R.J. (2000) Molecular and genetic analyses of the silky1 gene reveal conservation in floral organ specification between eudicots and monocots. 
Mol. Cell 5, 569-579.

20. Whipple, C.J., Ciceri, P., Padilla, C.M., Ambrose, B.A., Bandong, S.L., and Schmidt, R.J. (2004) Conservation of Bclass floral homeotic gene function between maize and Arabidopsis. Development 131, 6083-6091.

21. Yamaguchi, T., Lee, D.Y., Miyao, A., Hirochika, H., An, G., and Hirano, H.Y. (2006) Functional diversification of the two C-class MADS-box genes OSMADS3 and OSMADS58 in Oryza sativa. Plant Cell 18, 15-28. Angenent, G.C. and Colombo, L. (1996) Molecular control of ovule development. Trends Plant Sci. 1, $228-232$. require SEPALLATA MADS-box genes. Nature 405, 200-203.

24. Ditta, G., Pinyopich, A., Robles, P., Pelaz, S., and Yanofsky, M.F. (2004) The SEP4 gene of Arabidopsis thaliana functions in floral organ and meristem identity. Curr. Biol. 14, 1935-1940. Theissen, G. and Saedler, H. (2001) Floral quartets. Nature 409, 469-471.

26. Zahn, L.M., Leebens-Mack, J., DePamphilis, C.W., Ma, H., and Theissen, G. (2005) To B or not to B a flower: the role of DEFICIENS and GLOBOSA orthologs in the evolution of the angiosperms. J. Hered. 96, 225-240.

Curr. Opin. Genet. Dev. 15, 454-460.

28. Gill, D.E. (1989) Fruiting failure, pollinator inefficiency, and speciation in orchids. In Speciation and its Consequences. Otte, D. and Endler, J.A., Eds. Sinauer Associates, Sunderland, MA. pp. 458-481.

29. Darwin, C. (1885) On the Various Contrivances by Which Orchids are Fertilised by Insects. 2nd ed. John Murray, London.

Brown, R. (1810) Prodromus Florae Novae Hollandiae et Insula Van-Diemen. J. Johnson, London. gynostemium and labellum of orchids and other lilioid monocots. Biol. Rev. 77, 403-441. $\mathrm{Yu}, \mathrm{H}$. and Goh, C.J. (2000) Identification and characterization of three orchid MADS-box genes of the AP1/AGL9 subfamily during floral transition. Plant Physiol. 123, 1325-1336.

33. Skipper, M., Pedersen, K.B., Johansen, L.B., Frederiksen, S., Irish, V.F., and Johansen, B.B. (2005) Identification and quantification of expression levels of three FRUITFULL-like MADS-box genes from the orchid Dendrobium thyrsiflorum (Reichb. f.). Plant Sci. 169, 579-586.

34. Litt, A. and Irish, V.F. (2003) Duplication and diversification in the APETALA1/FRUITFULL floral homeotic gene lineage: implications for the evolution of floral development. Genetics 165, 821-833.

35. Hsu, H.F., Huang, C.H., Chou, L.T., and Yang, C.H. (2003) Ectopic expression of an orchid (Oncidium Gower Ramsey) AGL6-like gene promotes flowering by activating flowering time genes in Arabidopsis thaliana. Plant Cell Physiol. 44, 783-794.

36. Lee, S., Jeon, J.-S., An, K., Moon, Y.-H., Lee, S., Chung, Y.-Y., and An, G. (2003) Alteration of floral organ identity in rice through ectopic expression of OsMADS16. Planta 217, 904-911.

37. Nagasawa, N., Miyoshi, M., Sano, Y., Satoh, H., Hirano, H., Sakai, H., and Nagato, Y. (2003) SUPERWOMAN1 and DROOPING LEAF genes control floral organ identity in rice. Development 130, 705-718.

38. van Tunen, A.J., Eikelboom, W., and Angenent, G.C. (1993) Floral organogenesis in Tulipa. Flowering Newsl. 16, 33-37.

39. Baum, D.A. and Whitlock, B.A. (1999) Plant development: genetic clues to petal evolution. Curr. Biol. 9, R525R527.

40. Hsu, H.F. and Yang, C.H. (2002) An orchid (Oncidium Gower Ramsey) AP3-like MADS gene regulates floral formation and initiation. Plant Cell Physiol. 43, 1198-1209.

41. Tsai, W.C., Chuang, M.H., Kuoh, C.S., Chen, W.H., and Chen. H.H. (2004) Four DEF-like MADS box genes displayed distinct floral morphogenetic roles in Phalaenopsis orchid. Plant Cell Physiol. 45, 831-844.

42. Tsai, W.C., Lee, P.F., Chen, H.I., Hsiao, Y.Y., Wei, W.J., Pan, Z.J., Chuang, M.H., Kuoh, C.S., Chen, W.H., and Chen, H.H. (2005) PeMADS6, a GLOBOSA/PISTILLATA-like gene in Phalaenopsis equestris involved in petaloid formation, and correlated with flower longevity and ovary development. Plant Cell Physiol. 46, 1125-1139.

43. Xu, Y., Teo, L.L., Zhou, J., Kumar, P.P., and Yu, H. (2006) Floral organ identity genes in the orchid Dendrobium crumenatum. Plant J. 46, 54-68.

44. Ohno, S. (1970) Evolution by Gene Duplication. Springer, Berlin.

Cubas, P. (2004) Floral zygomorphy, the recurring evolution of a successful trait. BioEssays 26, 1175-1184. Dressler, R.L. (1993) Phylogeny and Classification of the Orchid Family. Cambridge University Press, Cambridge. Song, I.-J., Nakamura, T., Fukuda, T., Yokoyama, J., Ito, T., Ichikawa, H., Horikawa, Y., Kameya, T., and Kanno, A. (2006) Spatiotemporal expression of duplicate AGAMOUS orthologues during floral development in Phalaenopsis. Dev. Genes Evol. 4, 1-13.

48. Skipper, M., Johansen, L.B., Pederson, K.B., Frederiksen, S., and Johansen, B.B. (2006) Cloning and transcription analysis of an AGAMOUS- and SEEDSTICK ortholog in the orchid Dendrobium thyrsiflorum (Reichb. f.). Gene 366, 266-274.

49. Kim, S., Koh, J., Yoo, M.J., Kong, H., Hu, Y., Ma, H., Soltis, P.S., and Soltis, D.E. (2005) Expression of floral MADS-box genes in basal angiosperms: implications for the evolution of floral regulators. Plant J. 43, 724-744.

50. Kaufmann, K., Melzer, R., and Theissen, G. (2005) MIKC-type MADS-domain proteins: structure modularity, protein interactions and network evolution in land plants. Gene 347, 183-198. 
51. Honma, T. and Goto, K. (2001) Complexes of MADS-box proteins are sufficient to convert leaves into floral organs. Nature 409, 525-529.

52. Lu, Z.X., Wu, M., Loh, C.S., Yeong, C.Y., and Goh, C.J. (1993) Nucleotide sequence of a flower-specific MADS box cDNA clone from orchid. Plant Mol. Biol. 23, 901-904.

53. Cozzolino, S. and Widmer, A. (2005) Orchid diversity: an evolutionary consequence of deception? Trends Ecol. Evol. 20, 487-494.

54. Tsai, W.C., Hsiao, Y.Y., Lee, S.H., Tung, C.W., Wang, D.P., Wang, H.C., Chen, W.H., and Chen, H.H. (2006) Expression analysis of the ESTs derived from the flower buds of Phalaenopsis equestris. Plant Sci. 170, 426-432.

55. Wang, H.C., Kuo, H.C., Chen, H.H., Hsiao Y.Y., and Tsai, W.C. (2005) KSPF: using gene sequence patterns and data mining for biological knowledge management. Expert Syst. Appl. 28, 537-545.

56. Hsiao, Y.Y., Tsai, W.C., Kuoh, C.S., Huang, T.H., Wang, H.C., Wu, T.S., Leu, Y.L., Chen, W.H., and Chen, H.H. (2006) Comparison of transcripts in Phalaenopsis bellina and Phalaenopsis equestris (Orchidaceae) flowers to deduce monoterpene biosynthesis pathway. BMC Plant Biol., In Press.

This article should be cited as follows:

Tsai, W.-C. and Chen, H.-H. (2006) The orchid MADS-box genes controlling floral morphogenesis. TSW Development \& Embryology 1, 109-120. DOI 10.1100/tswde.2006.167.

\section{BIOSKETCH}

Hong-Hwa Chen is a professor in the Department of Life Sciences at National Cheng Kung University, and Wen-Chieh Tsai is currently a postdoctoral researcher in Dr. Chen's lab. They have worked on Phalaenopsis orchids for 10 years. They apply genomics approaches to study the nonmodel plants with large genome size by establishing EST databases, and focus on the B-class genes for orchid floral development. In addition, they have collaborated with colleagues on Chemistry and Information Management to reveal the orchid fragrance metabolic pathways. 